\title{
A 5.8 GHz Mixer using SiGe HBT Process
}

\author{
Sang-Heung Lee, Ja-Yol Lee, Seung-Yun Lee, Chan Woo Park, Sang Hoon Kim, Hyun-Chul Bae, \\ Jin-Yeong Kang, and Kyoung-Ik Cho
}

SiGe Devices Team, Electronics and Telecommunications Research Institute, Republic of Korea

\begin{abstract}
DSRC provides high speed radio link between Road Side Equipment and On-Board Equipment within the narrow communication area. In this paper, a 5.8 GHz down-conversion mixer for DSRC communication system is designed and fabricated using $0.8 \mu \mathrm{m}$ SiGe HBT process technology and $\mathrm{RF} / \mathrm{LO}$ matching circuits, $\mathrm{RF} / \mathrm{LO}$ input balun circuits and IF output balun circuit are all integrated on chip. The measured performance is $7.5 \mathrm{~dB}$ conversion gain, $-2.5 \mathrm{dBm}$ input IP3, $46 \mathrm{~dB}$ LO to RF isolation, $56 \mathrm{~dB}$ LO to IF isolation, current consumption of $21 \mathrm{~mA}$ for 3.0 $\mathrm{V}$ supply voltage and the chip size of fabricated mixer is $1.9 \mathrm{~mm} \times 1.3 \mathrm{~mm}$.
\end{abstract}

\section{INTRODUCTION}

ITS (Intelligent Transportation Systems) technology has been developed to solve the traffic problem such as traffic congestion and accidents and reduce the overall congestion cost. The final goal of ITS will be to improve the traffic efficiency and mobile safety without new road construction. DSRC (Dedicated Short Range Communication) provides high speed radio link between Road Side Equipment (RSE) and On-Board Equipment (OBE) within the narrow communication area. DSRC communication system has been developed worldwide and recently applied for Electronic Toll Collection (ETC), but most of all ITS services will be provided by DSRC communication [1].

The SiGe hetero-junction bipolar transistor (HBT) has been considered to be more suitable for RF integrated circuits than the $\mathrm{Si}$ bipolar junction transistor (BJT) because its electrical properties, such as current gain, power consumption, small-signal unity-gain frequency, and maximum oscillation frequency are superior to those of the Si BJT [2]. Because of having many advantages for those RF applications, SiGe HBT device has been adopted as a good candidate for manufacturing RF components such as LNAs, Mixers, VCOs, PLLs, RF transceivers.

Down-conversion mixers, which convert radio frequency (RF) signal to intermediate frequency (IF) signal, are very important building blocks within a radio system. Their performance affects the performance requirements of the entire system and the performance requirements of other building blocks.

In this paper, a $5.8 \mathrm{GHz}$ down-conversion mixer including RF/LO matching circuits, RF/LO input balun circuits and IF output balun circuit for DSRC receiver is designed and fabricated on chip, using SiGe HBT process technology of Electronics and Telecommunications Research Institute (ETRI). The measured performance was $7.5 \mathrm{~dB}$ conversion power gain, $-2.5 \mathrm{dBm}$ input IP3, $46 \mathrm{~dB}$ LO to RF isolation, 56 $\mathrm{dB}$ LO to IF isolation, current consumption of $21 \mathrm{~mA}$ for $3.0 \mathrm{~V}$ supply voltage.

\section{SIGE HBT DC AND AC CHARACTERISTICS}

Electrical performance parameters of SiGe HBT such as cut-off frequency $\left(f_{\mathrm{T}}\right)$, maximum oscillation frequency $\left(f_{\max }\right)$, minimum noise figure $\left(\mathrm{NF}_{\min }\right)$ are critically dependent on not only the base thickness, but also the fabrication process. We used RPCVD system to grow base epitaxial layer, and adopted LOCOS isolation to separate device and device terminals. The maximum values of $f_{\mathrm{T}} / f_{\max }$ were $41 \mathrm{GHz} / 42 \mathrm{GHz}$ at $\mathrm{V}_{\mathrm{CE}}=2 \mathrm{~V}, \mathrm{I}_{\mathrm{C}}=$ $1.83 \mathrm{~mA}$ as shown in Fig. 1. In Table 1, the main DC and AC parameters of HBT with emitter area of $0.5 \times 6.0$ $\mu \mathrm{m}^{2}$ are summarized. Passive elements used in this paper were MIM capacitor, parallel-branch spiral inductor, and resistors composed of metal, emitter poly-silicon, and base poly-silicon.

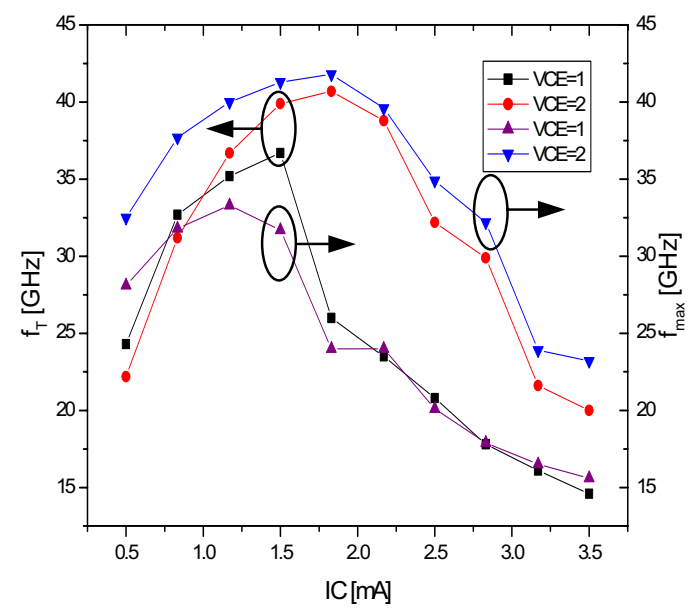

Fig. 1. AC characteristics of SiGe HBT

\section{SIGE HBT MIXER DESIGN}

The architectures of DSRC RF front-end receiver and down-conversion mixer in this receiver are shown in Fig. 2. The LNA output and LO output signals go RF single input port and LO single input port of mixer, respectively, and single output signal of mixer goes IF input port. The $5.81 \mathrm{GHz}$ single-ended RF input and the 5.495 singleended LO input are converted to be differential to feed in the double balanced mixer by use of on-chip active balun. 
Fig. 3 shows the single-input, balanced-output differential balun, which is used as the RF and LO balun. In this Fig. 3, the single input is applied to the base of transistor Q1, and the output is measured between the two collectors, which are at the same dc potential. The gain of balun is determined by transconductances of Q1, Q2, the degeneration inductors L1, L2, and the output resistors R1, R2. The amount of the LO suppression is determined by the common-mode gain of the balun, and the differential-type balun rejects very well the commonmode gain. The higher the output impedance of the current source, the better the LO suppression [3,4]. The capacitor $\mathrm{C} 1$ separates $\mathrm{DC}$ bias from ground.

Table 1. SiGe HBT DC \& AC Characteristics

\begin{tabular}{l|c}
\hline Emitter area $\left[\mu \mathrm{m}^{2}\right]$ & $0.5 \times 6.0$ \\
\hline $\mathrm{DC}$ current gain & 296 \\
\hline $\mathrm{BV}_{\mathrm{EBO}}[\mathrm{V}]$ & 0.95 \\
\hline $\mathrm{BV}_{\mathrm{CBO}}[\mathrm{V}]$ & 10.7 \\
\hline $\mathrm{BV}_{\mathrm{CEO}}[\mathrm{V}]$ & 3.5 \\
\hline $\mathrm{R}_{\mathrm{C}}[\Omega]$ & 61 \\
\hline $\mathrm{R}_{\mathrm{E}}[\Omega]$ & 30 \\
\hline$f_{\mathrm{T}}[\mathrm{GHz}]$ & 41 \\
\hline$f_{\max }[\mathrm{GHz}]$ & 42
\end{tabular}

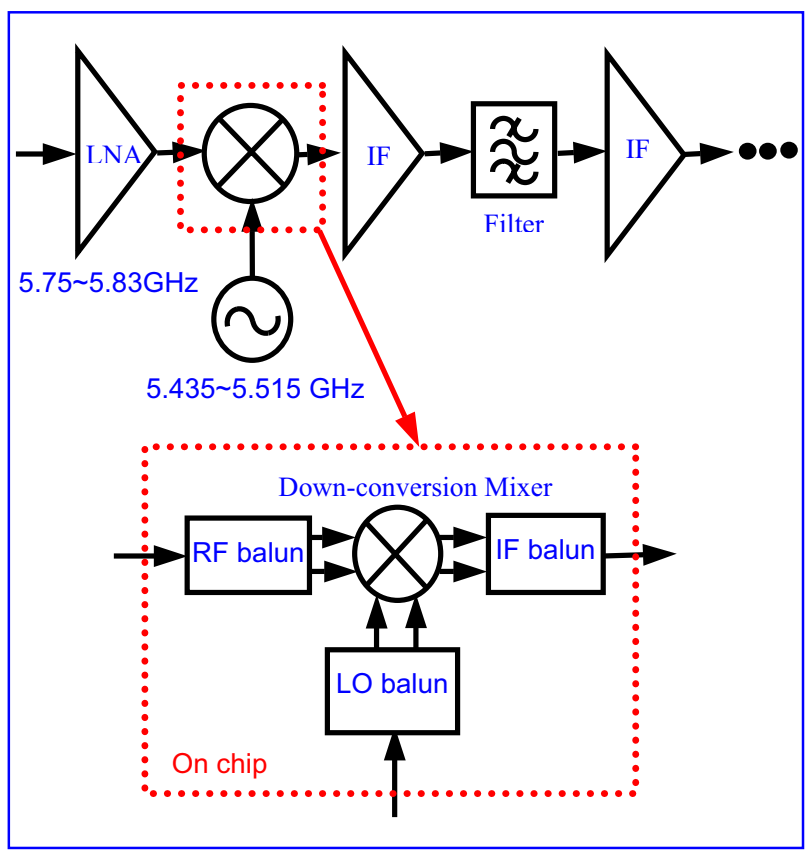

Fig. 2. Architecture of DRSC RF receiver and mixer

Fig. 4 shows a push-pull balun which is composed of a common-emitter with degeneration and the commoncollector. The degeneration resistor R3 controls the gain of the common emitter path for the same amount of gain for both inverting and non-inverting signals, resulting in maximum cancellation of LO leakage at the output of the balun. R4 is included for output impedance matching. The gain of the balun is the sum of the two signal path gains [5]. The common-collector path provides highly linear performance and the common-emitter is more linear than the differential-pair for the same bias current and transconductance [4].

The above baluns are deigned and all integrated on chip together with mixer core circuit and provide good isolation.

Double-balanced bipolar mixer in the form of Gilbert cell used in this paper is shown in Fig. 5, which is differential structure to avoid the common-mode noise. When the switching transistors of the Gilbert cell mixer are driven fully differentially, theoretically, there is no LO leakage at the output. The emitter inductive degeneration (L3, L4) is also added to improve linearity.

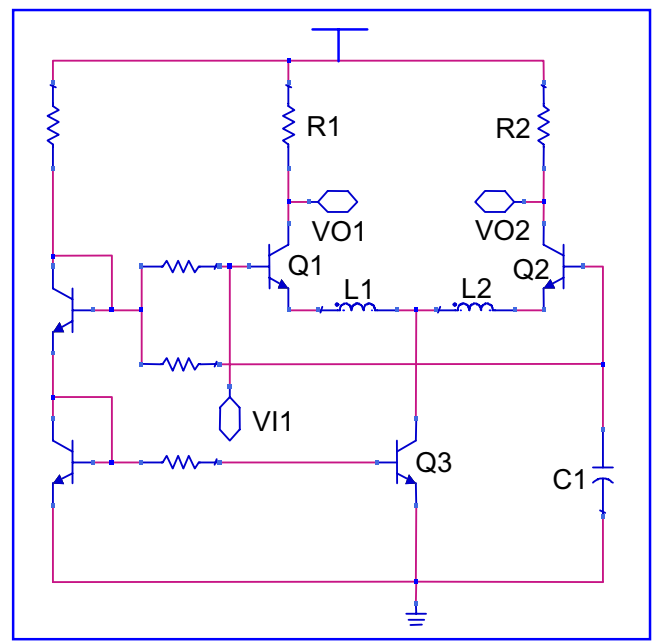

Fig. 3. RF/LO input balun with bias circuit

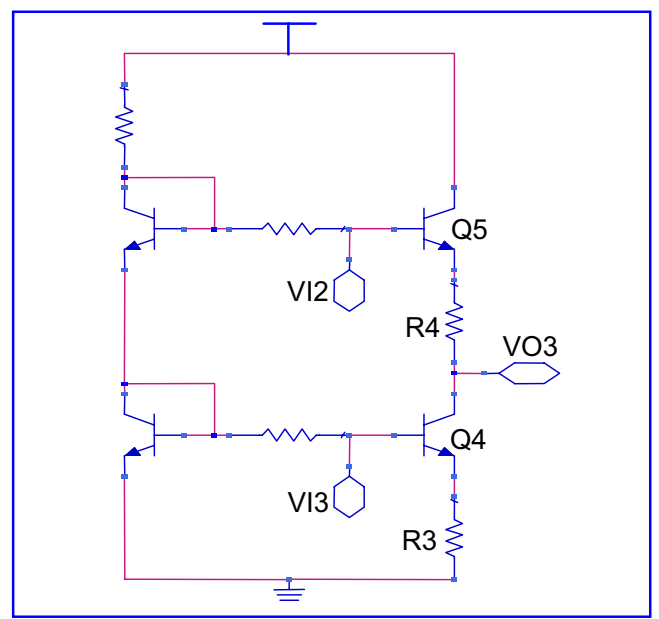

Fig. 4. IF output balun with bias circuit

\section{FABRICATION AND MEASUREMENTS}

We designed and fabricated the mixer circuit using 0.8 $\mu \mathrm{m}$ SiGe HBT process technology. The manufactured mixer photograph is shown in Fig. 6 and this chip size is $1.9 \mathrm{~mm} \times 1.3 \mathrm{~mm}$. In Fig. 6, the upper pad is for supply voltage. The left, lower and right center pads are LO input, RF input, IF output pads, respectively.

For this measurement, two RF power sources HP83650B, HP83752B, and a spectrum analyzer HP8563E were used. The measurement conditions of the fabricated mixer were $\mathrm{RF}=-30 \mathrm{dBm}, \mathrm{LO}=-5.0 \mathrm{dBm}$, $\mathrm{VCC}=3.0 \mathrm{~V}$ (with local biases $\mathrm{VA}=3.0$ and $\mathrm{VB}=2.5$ ). Fig. 
7 shows the measured frequency spectrum at IF output port (upper figure: $50 \mathrm{MHz} \sim 8.0 \mathrm{GHz}$ range, lower figure: $100 \mathrm{MHz}$ span) and conversion gain is $7.5 \mathrm{~dB}$, $\mathrm{LO}$ to RF port-to-port isolation is $46 \mathrm{~dB}$, LO to IF portto-port isolation is $56 \mathrm{~dB}$. Fig. 8 shows the measured IP3 when the LO input power was fixed to $-5.0 \mathrm{dBm}$ and the $\mathrm{RF}$ input power was swept from $-30 \mathrm{dBm}$ to $-3 \mathrm{dBm}$ and from this figure, IIP 3 is $-2.5 \mathrm{dBm}$. Table 2 shows comparison of the simulated and measured results.

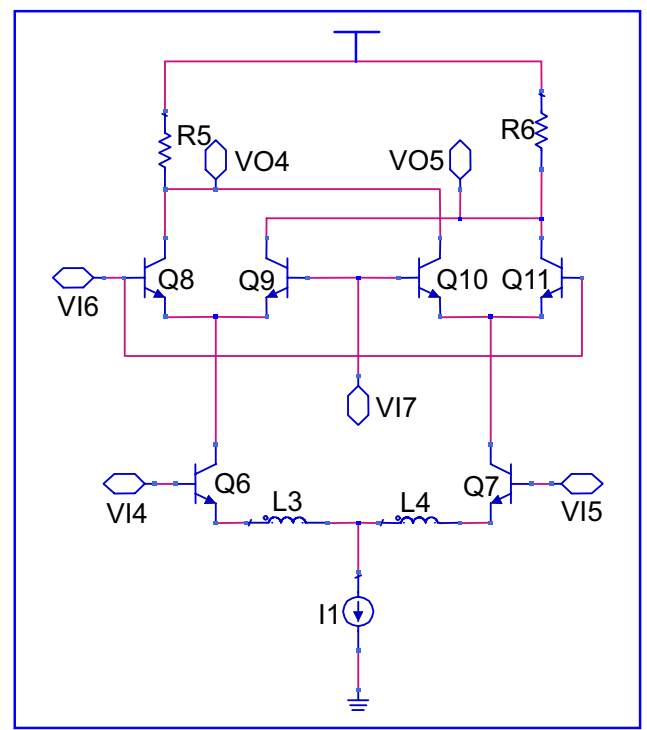

Fig. 5. Mixer with emitter inductive degeneration

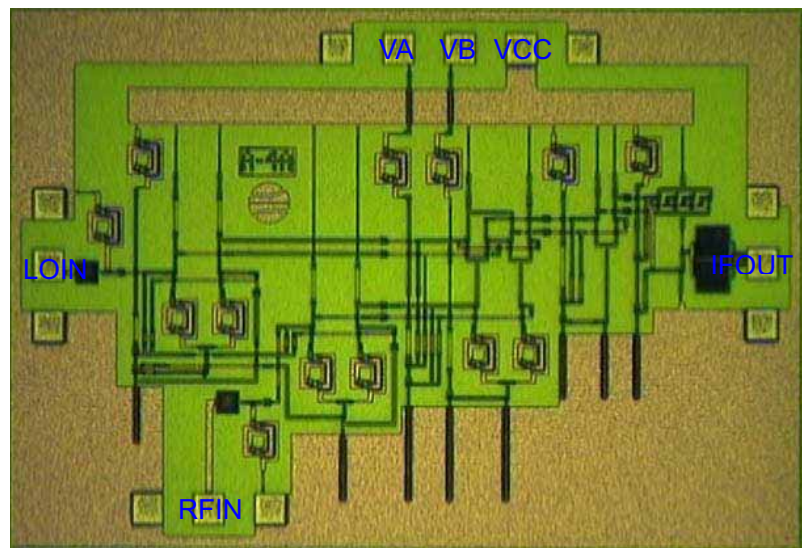

Fig. 6. Photograph of the fabricated mixer

Table 2. The performance of fabricated mixer

\begin{tabular}{l|c|c}
\hline \multicolumn{1}{c|}{ Parameters } & Simulated & Measured \\
\hline RF frequency [GHz] & 5.810 & 5.810 \\
\hline LO frequency [GHz] & 5.495 & 5.495 \\
\hline Conversion gain [dB] & 8.0 & 7.5 \\
\hline LO to RF isolation [dB] & 68 & 46 \\
\hline LO to IF isolation [dB] & 43 & 56 \\
\hline RF to LO isolation [dB] & 56 & 34 \\
\hline RF to IF isolation [dB] & 40 & 30 \\
\hline IIP3 [dBm] & 1.5 & -2.5 \\
\hline IC [mA] & 20 & 21 \\
\hline
\end{tabular}

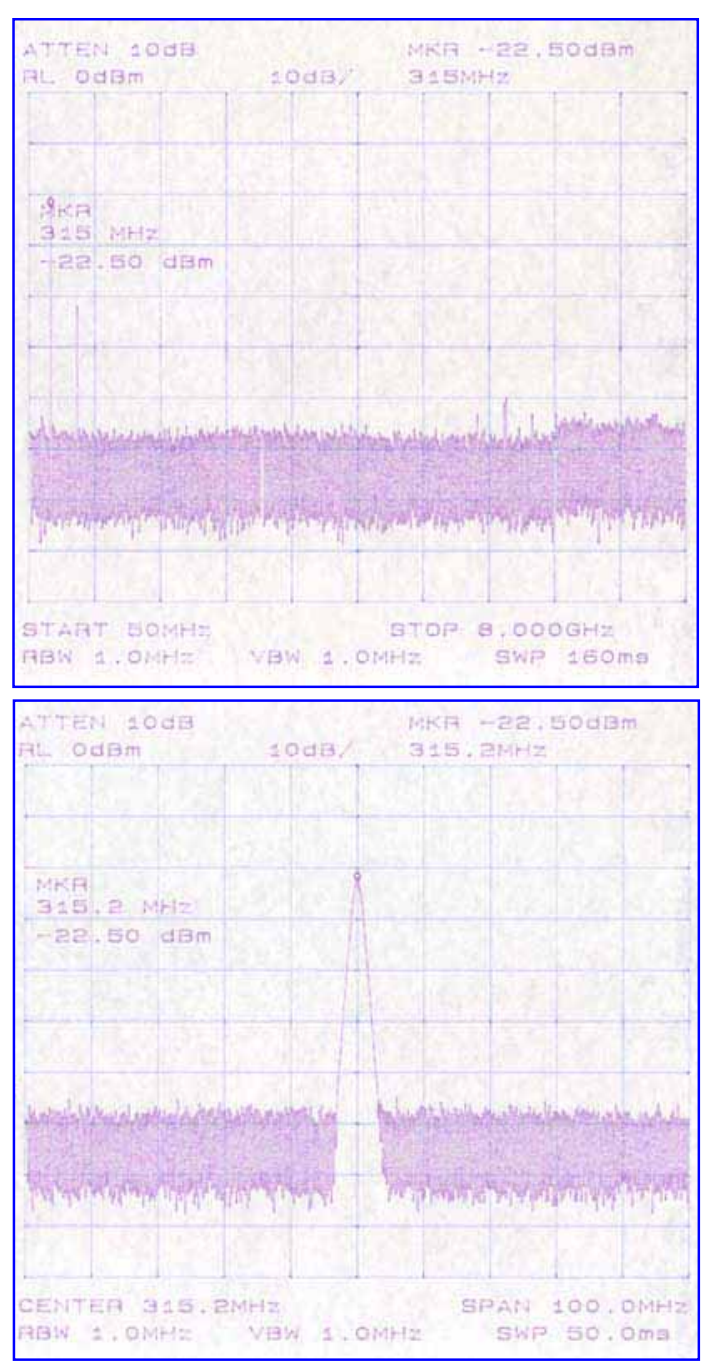

Fig. 7. The measured frequency spectrum at IF port (upper: $50 \mathrm{MHz} 8.0 \mathrm{GHz}$ range, lower: $100 \mathrm{MHz}$ span)

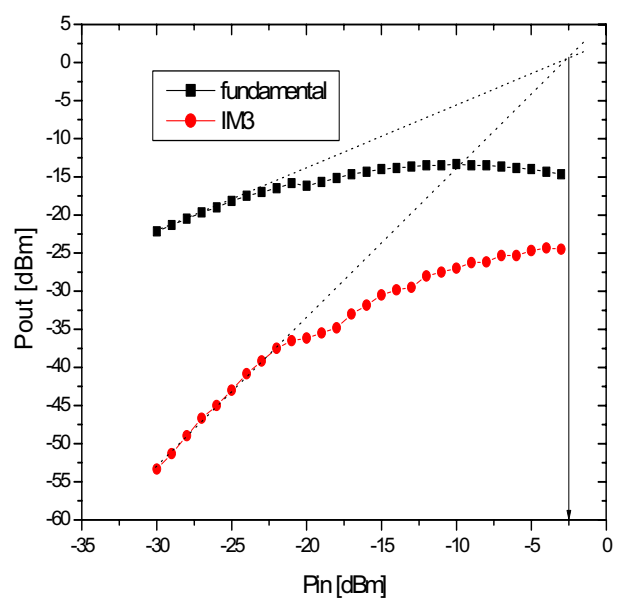

Fig. 8. The measured IP3 characteristics

\section{CONCLUSION}

DSRC provides high speed radio link between Road Side Equipment (RSE) and On-Board Equipment (OBE) within the narrow communication area and most of all ITS services will be provided by DSRC communication. In this paper, a $5.8 \mathrm{GHz}$ down-conversion mixer for 
DSRC receiver was designed and fabricated using 0.8 $\mu \mathrm{m}$ SiGe HBT process technology and RF/LO matching circuits, RF/LO input balun circuits and IF output balun circuit were all integrated on chip. The measured performance was $7.5 \mathrm{~dB}$ conversion power gain, -2.5 $\mathrm{dBm}$ input IP3, $46 \mathrm{~dB}$ LO to RF isolation, $56 \mathrm{~dB}$ LO to IF isolation, current consumption of $21 \mathrm{~mA}$ for $3.0 \mathrm{~V}$ supply voltage.

\section{ACKNOWLEDGMENTS}

This work was supported by the Ministry of Information and Communication, Republic of Korea.

\section{REFERENCES}

[1] H. Oh, C. Yae, D. Ahn, and H. Cho, "5.8 GHz DSRC Packet Communication System for ITS Services," IEEE Vehicular Technology Conference, pp. 2223-227, Fall 1999.
[2] S.-Y. Lee, H.-S. Kim, S.-H. Lee, K.-H Shim, J.-Y. Kang, M.-K. Song, "The behavior of Ti silicidation on $\mathrm{Si} / \mathrm{SiGe} / \mathrm{Si}$ base and its effect on base resistance and fmax in SiGe hetero-junction bipolar transistors," Journal of Materials Science: Materials in Electronics, vol. 12, no. 8, pp. 467-472, August 2001.

[3] R.A. Gayakwad, OP-Amps and Linear Integrated Circuits, Prentice-Hall, Inc., 1988.

[4] K.L. Fong and R.G. Meyer, "High-Frequency Nonlinearity Analysis of Common-Emitter and Differential-Pair Transconductance Stages," IEEE Journal of Solid-State Circuits, vol. 33, pp. 548-555, April 1998.

[5] D.-Y. Kim, S.-G. Lee, J.-H. Lee, "Up-Conversion Mixer for PCS Application using Si BJT,” IEEE 2nd International Conference on Microwave and Millimeter Wave Technology Proceedings, pp. 424427, 2000 . 